\title{
N-Acetylcysteine in the Prevention of Radiocontrast-Induced Nephropathy: Clinical Trials and End Points
}

\author{
Ute Hoffmann ${ }^{a}$ Bernhard Banas ${ }^{a}$ Michael Fischereder ${ }^{b}$ \\ Bernhard K. Krämera \\ aKlinik und Poliklinik für Innere Medizin II, Klinikum der Universität Regensburg, Regensburg, und \\ ${ }^{b}$ Medizinische Poliklinik Innenstadt, Klinikum der Ludwig-Maximilians-Universität, München, Deutschland
}

\section{Key Words \\ $\mathrm{N}$-acetylcysteine $\cdot$ Radiocontrast-induced nephropathy . Creatinine $\cdot$ Cystatin $\mathrm{C}$. Glomerular filtration rate}

\begin{abstract}
$\mathrm{N}$-acetylcysteine (NAC) has been suggested to prevent radiocontrast-induced nephropathy (RCIN) in patients with a reduced renal function. However, clinical studies have not been demonstrating this effect consistently. Also, reviews and meta-analyses dealing with the question of prevention of RCIN by NAC have been controversial. Nearly all investigators used serum creatinine as surrogate end point of their trials, and changes in serum creatinine concentrations are thought to reflect the extent of renal injury as primary outcome. In a recent study, an effect of NAC on creatinine values and estimated glomerular filtration rate without any effect on cystatin $C$ levels has been shown in volunteers with a normal renal function. Therefore, before renal protective effects of NAC in RCIN are proposed, any direct effects of NAC on creatinine, urea, and estimated glomerular filtration rate
\end{abstract}

Presented at NephroPharmacology 7 meeting, October 10, 2003, Ulm, Germany.

\section{KARGER}

Fax +41613061234

E-Mail karger@karger.ch

www. karger.com
(C) 2004 S. Karger AG, Basel

1420-4096/04/0273-0161\$21.00/0

Accessible online at:

www. karger.com/kbr should be addressed. In future trials, the glomerular filtration rate should preferentially be measured directly, or at least additional markers of the renal function (e.g., serum cystatin C) have to be assessed. Furthermore, additional 'hard' end points, i.e., hospital morbidity, mortality, or dialysis dependency, should be considered in the design of future studies of RCIN.

Copyright @ 2004 S. Karger AG, Basel

\section{Introduction}

Radiocontrast-induced nephropathy (RCIN) is defined as a sudden decline in renal function occurring after exposure to intravenous radiographic contrast agents that is not attributable to other causes. Typically, the serum creatinine level begins to increase $24-72 \mathrm{~h}$ after administration of contrast medium, peaks at 3-5 days, and requires further 3-5 days to return to baseline. In most studies, an acute radiocontrast-agent-induced reduction in the renal function was defined as an increase in the serum creatinine concentration of at least $0.5 \mathrm{mg} / \mathrm{dl}$ within $48 \mathrm{~h}$ after the administration of contrast agents.

Although several approaches have been explored to prevent renal contrast damage, RCIN continues to be a serious complication in patients with preexisting renal

Ute Hoffmann, MD, Klinik und Poliklinik für Innere Medizin II

Klinikum der Universität Regensburg

DE-93042 Regensburg (Germany)

Tel. +49 9419447301 , Fax +499419447302

E-Mail Ute.Hoffmann@klinik.uni-regensburg.de 
insufficiency. Effective regimens are still lacking, as the pathogenesis of radiocontrast-induced renal dysfunction is only partially understood.

Presently, hydration of the patient [1-3] and the use of small amounts of low-osmolar, nonionic contrast agents [4] are recommended. RCIN may be less likely to develop in high-risk patients, when iso-osmolar contrast medium is used rather than low-osmolar contrast medium [5]; however, further studies in support of this assumption are needed. Evidence from clinical trials did not sufficiently prove a beneficial effect of hemodialysis/hemofiltration or theophylline, aminophylline, prostaglandins, diuretics, dopamine, calcium channel blockers, atrial natriuretic peptides, endothelin receptor blockers, or mannitol to recommend the general use of one of these therapies or compounds [6-8].

The administration of N-acetylcysteine (NAC) seems to be an additional option to avoid renal injury, even if there are conflicting data about the additive protective properties of NAC when used in conjunction with hydration [9-19]. Interestingly, the advantage of NAC in most studies was based on a decrease in the serum creatinine concentration in patients exposed to contrast agents plus NAC, while often an unchanged serum creatinine concentration was observed after radiocontrast exposure in patients without NAC. In a recent study [20], we have shown that NAC led to a decrease in serum creatinine, while the serum cystatin $\mathrm{C}$ concentration did not change in volunteers with a normal renal function.

Thus, in the present review, we will first discuss the available evidence that NAC is able to prevent RCIN and secondly question whether the sole measurement of serum creatinine suffices as a reliable marker of the renal function in this setting.

\section{N-Acetylcysteine}

NAC is a thiol-containing compound which stimulates the intracellular synthesis of glutathione and enhances the glutathione S-transferase activity [21, 22]. NAC is supposed to have antioxidant properties because it suppresses plasma and tissue angiotensin-converting enzyme activities [23], attenuates cytotoxic properties of advanced glycation end products [24], and decreases homocysteine plasma levels [25]. NAC is demonstrated to act as an antioxidant only in oxidative stress conditions [26]. Some reports have shown that it can inhibit nuclear factor kappa $\mathrm{B}$ activation in renal mesangial and epithelial cells $[27,28]$.
In animal studies, nephroprotective properties of NAC have been demonstrated in ciclosporin-induced nephrotoxicity as well as in ischemia/reperfusion injury [29, 30]. In contrast, no benefits of NAC administration were found in a model of renal interstitial inflammation [31]. Moreover, at doses of $1.2 \mathrm{~g}$ daily, NAC may even exert pro-oxidative properties in subjects with normal intracellular glutathione levels [32].

By scavenging free radicals produced after contrast media administration, renal toxicity might be prevented by NAC [33]. The findings of Heyman et al. [34] in rats suggest that NAC-related protective properties during the evolution of acute renal failure may be mediated in part by dilation of the constricted renal vasculature. Previous studies already have described vasodilatory properties of NAC $[34,35]$.

\section{NAC and RCIN}

Although the concept of prevention of RCIN by NAC is favored by its simplicity and low cost, the role of NAC for the prophylaxis of RCIN has not been established definitively. To date, unfortunately only three trials [11, $14,15]$ described the effects of NAC not only on serum creatinine but also on clinical end points. Kay et al. [11] investigated the effect of NAC on the length of hospital stay as a secondary end point and found a significant reduction of $12 \mathrm{~h}$ in patients given NAC. In another prospective, randomized, double-blind, and placebo-controlled study designed to evaluate the efficacy and safety of NAC in the prevention of RCIN in patients undergoing coronary angiography [14], there was no difference in length of stay, hospital charges, or serum creatinine changes between the NAC-treated and the control group. Goldenberg et al. [15] reported similar findings in patients with chronic renal insufficiency undergoing cardiac catheterization. The incidence of in-hospital adverse clinical events and the length of hospital stay did not differ between the NAC-treated and the control groups, and there were no differences in creatinine levels between the treatment groups.

In all other studies, due to the rare occurrence of severe clinical adverse events after radiocontrast administration, only surrogate markers of a reduced glomerular filtration rate (GFR), such as creatinine or urea, have been used as primary outcome, and renal injury has been accordingly extrapolated from changes in serum chemistry. This constitutes a major limitation of such studies. When reviewing the published studies to date, one has to keep in mind 
that publication bias may well be present and that the comparability between trials is limited due to differences in baseline characteristics of the patients and in major study design details, particularly the severity of chronic renal insufficiency before the procedure and the amount of radiocontrast media.

Several meta-analyses of the presently available data concerning the role of NAC in the prevention of RCIN have been published during the last months. Birck et al. [19] in their meta-analysis studied seven randomized controlled trials comparing orally given NAC and hydration with hydration alone for preventing RCIN in a total of 805 patients with chronic renal insufficiency. The common end point of all these studies was a change in the serum creatinine concentration. A rise in serum creatinine $>0.5 \mathrm{mg} / \mathrm{dl}$ was considered to indicate RCIN. Four studies [9-11, 36] showed a statistically significant reduction of the relative risk for the development of RCIN in patients given NAC, whereas the remaining three showed no significant benefit of preprocedural NAC [17, 18, 37]. By combining the effect sizes of these seven trials by a random-effects model, a significant relative risk reduction of 56\% was seen in patients given NAC. Alonso et al. [38] and Findlay and Dwomoa [39] described nearly similar findings when performing meta-analyses of both seven blinded and unblinded randomized controlled trials, but in both analyses, there was a significant heterogeneity of the effect of therapy. Another recent meta-analysis performed by Pannu et al. [40] found only a borderline statistical significance of the efficacy of NAC for preventing RCIN. However, in this analysis, the effect of NAC was not statistically significant in several prespecified subgroup analyses, and the results were not robust to the addition of hypothetical new or unidentified randomized trials. In a further recently published meta-analysis of eleven studies [41], no benefit of NAC in reducing the risk of RCIN in patients with baseline renal dysfunction was found.

In a new experimental study performed by Emch and Haller [42], a method for preventing renal tubular vacuolization by administration of NAC prior to contrast medium administration was tested in rats. The occurrence of renal tubular vacuolization in the NAC-treated groups was similar to that in control groups.

In contrast to all other studies assessing the effects of orally administered NAC, a recent study [43] compared intravenous NAC and hydration given immediately before coronary angiography/intervention with hydration alone for the prevention of RCIN. In the NAC-treated group, the mean serum creatinine concentration de- creased significantly from 1.85 to $1.77 \mathrm{mg} / \mathrm{dl} 48 \mathrm{~h}$ after contrast agent administration. In the group without NAC, the serum creatinine level increased slightly but not significantly. RCIN, defined in this study as an increase in the serum creatinine concentration by $25 \%$ occurred in 2 of the 41 patients in the NAC-treated group and in 8 of 39 patients in the group not receiving NAC $(p=0.045)$. After intravenous injection of NAC, however, $14.5 \%$ of the patients suffered flushing, itching, or rash.

Besides the question of the efficacy of NAC for RCIN prevention, it has to be asked whether the serum creatinine concentration is a reliable surrogate marker for the renal function at all. It is well known that the serum concentration of creatinine is determined not just by the GFR. Alterations in renal handling, i.e., tubular secretion and metabolism of creatinine, and methodological interference in its measurement may influence the serum concentration of creatinine. Due to dietary creatinine intake, tubular secretion of creatinine, and variations in the patient's muscle mass, the use of serum creatinine may inaccurately estimate the GFR.

Rickli et al. [44] recently compared serum cystatin C and serum creatinine to examine their kinetics after application of radiocontrast media in patients with a normal to decreased GFR. In this study, cystatin C achieved a maximum increase within $24 \mathrm{~h}$ after radiocontrast application, whereas serum creatinine started to increase at that time and continued to increase at $48 \mathrm{~h}$. Cystatin $\mathrm{C}$ is suggested to be a potential early marker of nephrotoxicity.

In a recent prospective study [20], we have studied the potential effects of NAC on serum creatinine, independent of alterations in the GFR. For this purpose, volunteers with a normal renal function who did not receive radiocontrast media were enrolled. NAC was given orally at a dose of $600 \mathrm{mg}$ every $12 \mathrm{~h}$ for a total of four doses. The serum levels of creatinine, urea, albumin, and cystatin $\mathrm{C}$ were determined before administration of NAC and 4 and $48 \mathrm{~h}$ after the last intake. Serum creatinine was measured both enzymatically and by the Jaffé method. The GFR was estimated (eGFR) on the basis of serum creatinine, urea, and albumin concentrations and weight, age, and sex, using the equation developed by Levey et al. [45]. There was a significant decrease of the mean serum creatinine concentration $(\mathrm{p}<0.05)$ and a significant increase of the eGFR $(\mathrm{p}<0.02) 4 \mathrm{~h}$ after the last administration of NAC, whereas the cystatin C concentration did not change significantly (fig. 1). The latter observation is not quite unexpected, as direct effects of NAC on the human renal function have not been reported yet. Taken together, two explanations for our findings are possible: 

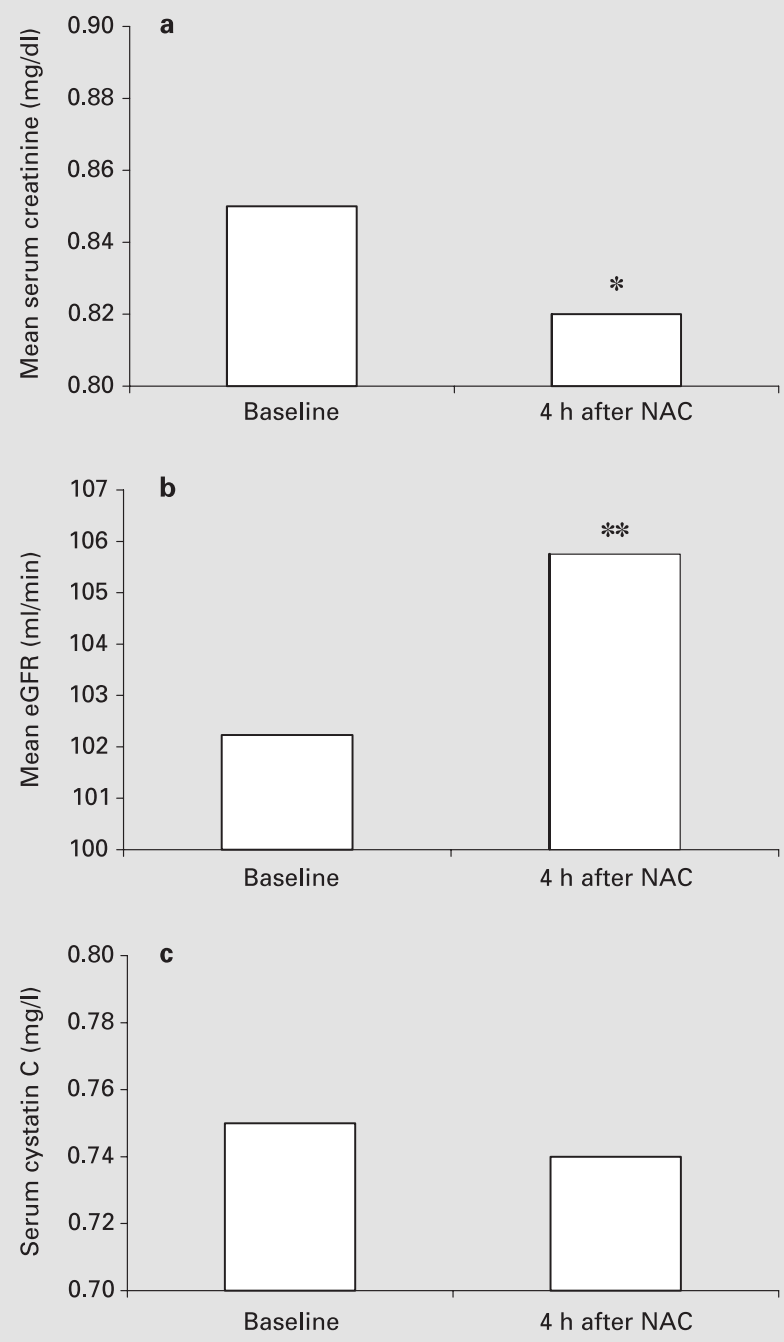

Fig. 1. Surrogate markers of the renal function. Mean serum creatinine (a), eGFR (b), and cystatin C (c) before (baseline) and $4 \mathrm{~h}$ after the last intake of NAC. There were a significant decrease of the mean serum creatinine concentration $(* \mathrm{p}<0.05)$ and a significant increase of the eGFR $(* * p<0.02) 4 \mathrm{~h}$ after the last administration of NAC, whereas the cystatin $\mathrm{C}$ concentration did not change significantly [20].

First, NAC truly improves the GFR, but cystatin C fails to detect such an improvement. This appears unlikely, since several studies comparing cystatin $\mathrm{C}$ and creatinine with the GFR employing the ${ }^{51} \mathrm{Cr}$-EDTA clearance, the gold standard to measure the GFR, documented that cystatin $\mathrm{C}$ determination is superior to creatinine measurement [46-49]. Furthermore, contrary to the serum creatinine concentration, the serum cystatin $\mathrm{C}$ level is independent of age, sex, and muscle mass.

Second, NAC does not alter the GFR, but causes a decrease in the serum creatinine concentration through another mechanism. A number of reasons favor this assumption. Creatinine is predominantly but not exclusively eliminated by glomerular filtration. Especially in patients with an impaired renal function, the extent of tubular secretion may contribute significantly to the total creatinine excretion. Furthermore, the creatinine metabolism is affected by NAC either through direct activation of creatinine kinase or through reversal of inhibition by free radicals [50].

Although these data were obtained in healthy adults, it is likely that the underlying physiological mechanisms are the same in individuals with renal disease. In contrast, the effects of NAC on renal tubular creatinine secretion or muscle metabolism may be even more prominent in such patients. However, to prove the same effect in patients with an impaired renal function, further studies using a similar protocol without administration of contrast agents have to be done. Nevertheless, the data obtained in our study clearly cast some doubt on the present practice to administer NAC for protection from RCIN.

\section{Conclusions}

Efficacious and safe prevention of RCIN is expected to decrease morbidity, including the need for dialysis, and mortality during hospitalization and thus should reduce health care costs. So far, only hydration and the use of lowosmolar contrast media proved to be beneficial. When the role of NAC in human RCIN is studied, special attention must be given to the end point used to determine presence or absence of renal injury. The presently available data from human studies as well as the meta-analyses represent a special dilemma, as they show conflicting results, even for the most common surrogate end point serum creatinine. In a recent study [20], we have shown that NAC leads to a decrease of serum creatinine, but that another surrogate marker of renal function, serum cystatin $\mathrm{C}$, does not change after NAC administration.

Therefore, it is suggested to assess hard clinical end points rather than solely changes in serum creatinine in future studies. If surrogate parameters of renal function are used to prove the protective effect of NAC, the study designs should preferably include direct GFR measurements or include at least another surrogate marker of the renal function, e.g., cystatin C. 
1 Murphy SW, Barrett BJ, Parfrey PS: Contrast nephropathy. J Am Soc Nephrol 2000;11:177182.

2 Trivedi HS, Moore H, Nasr S, Aggarwal K, Agrawal A, Goel P, Hewett J: A randomized prospective trial to assess the role of saline hydration on the development of contrast nephrotoxicity. Nephron Clin Pract 2003;93:c29-c34.

3 Solomon R, Werner C, Mann D, D'Elia J, Silva $\mathrm{P}$ : Effects of saline, mannitol, and furosemide to prevent acute decreases in renal function induced by radiocontrast agents. $\mathrm{N}$ Engl $\mathrm{J}$ Med 1994;331:1416-1420.

4 Barrett BJ, Carlisle EJ: Meta-analysis of the relative nephrotoxicity of high- and low-osmolality iodinated contrast media. Radiology 1993; 188:171-178.

5 Aspelin P, Aubry P, Fransson SG, Strasser R, Willenbrock R, Berg KJ: Nephrotoxic effects in high-risk patients undergoing angiography. N Engl J Med 2003;348:491-499.

6 Liebl R, Krämer BK: Komplikationen nach Applikation von Röntgen-Kontrastmitteln bei Risikopatienten: Ist eine prophylaktische Dialyse sinnvoll? Dtsch Med Wochenschr 1996; 121:1475-1479.

7 Marenzi G, Marana I, Lauri G, Assanelli E, Grazi M, Campodonico J, Trabattoni D, Fabbiocchi F, Montorsi P, Bartorelli AL: The prevention of radiocontrast-agent-induced nephropathy by hemofiltration. N Engl J Med 2003;349:1333-1340.

8 Krämer BK, Kammerl M, Schweda F, Schreiber M: A primer in radiocontrast-induced nephropathy. Nephrol Dial Transplant 1999;14: 2830-2834.

9 Tepel M, van der Giet M, Schwarzfeld C, Laufer U, Liermann D, Zidek W: Prevention of radiographic-contrast-agent-induced reductions in renal function by acetylcysteine. $\mathrm{N}$ Engl J Med 2000;343:180-184.

10 Shyu KG, Cheng JJ, Kuan P: Acetylcysteine protects against acute renal damage in patients with abnormal renal function undergoing a coronary procedure. J Am Coll Cardiol 2002;40: 1383-1388.

11 Kay J, Chow WH, Chan TM, Lo SK, Kwok OH, Yip A, Fan K, Lee CH, Lam WF: Acetylcysteine for prevention of acute deterioration of renal function following elective coronary angiography and intervention: A randomized controlled trial. JAMA 2003;289:553-558.

12 Diaz-Sandoval LJ, Kosowsky BD, Losordo DW: Acetylcysteine to prevent angiographyrelated renal tissue injury (the APART trial). Am J Cardiol 2002;89:356-358.

13 Tadros GM, Mouhayar EN, Akinwande AO, Campbell B, Wood C, Blankenship JA: Prevention of radiocontrast-induced nephropathy with $\mathrm{N}$-acetylcysteine in patients undergoing coronary angiography. J Invasive Cardiol 2003; $15: 311-314$

14 Oldemeyer JB, Biddle WP, Wurdeman RL, Mooss AN, Cichowski E, Hilleman DE: Acetylcysteine in the prevention of contrast-induced nephropathy after coronary angiography (abstract). Am Heart J 2003; 146:E23.
15 Goldenberg I, Jonas M, Matetzki S, Shechter M, Agranat O, Har-Zahav Y, Elian D, Guetta V: Contrast-associated nephropathy and clinical outcome of patients with chronic renal insufficiency undergoing cardiac catheterization: Lack of additive benefit of acetylcysteine to saline hydration (abstract). J Am Coll Cardiol 2003; 41:537A

16 Boccalandro F, Amhad M, Smalling RW, Sdringola S: Oral acetylcysteine does not protect renal function from moderate to high doses of intravenous radiographic contrast. Catheter Cardiovasc Interv 2003;58:336-341.

17 Briguori C, Manganelli F, Scarpato P, Elia PP, Golia B, Riviezzo G, Lepore S, Librera M, Villari B, Colombo A, Ricciardelli B: Acetylcysteine and contrast agent-associated nephrotoxicity. J Am Coll Cardiol 2002;40:298-303.

18 Durham JD, Caputo C, Dokko J, Zahrakis T, Pahlavan M, Keltz J, Dutka P, Marzo K, Maesaka JK, Fishbane S: A randomized controlled trial of $\mathrm{N}$-acetylcysteine to prevent contrast nephropathy in cardiac angiography. Kidney Int 2002;62:2202-2207.

19 Birck R, Krzossok S, Markowetz F, Schnülle P, van der Woude F, Braun C: Acetylcysteine for prevention of contrast nephropathy: Metaanalysis. Lancet 2003;362:598-603.

20 Hoffmann U, Fischereder M, Krüger B, Drobnik W, Krämer BK: The value of N-acetylcysteine in the prevention of radiocontrast-induced nephropathy seems questionable. J Am Soc Nephrol 2004;15:407-410.

21 Aruoma O, Hallowell B, Hoey B, Butler J: The antioxidant action of $\mathrm{N}$-acetylcysteine: Its reaction with hydrogen peroxide, hydroxyl radical, superoxide, and hypochlorous acid. Free Radic Biol Med 1989;6:593-597.

22 Shan Z, Tan D, Satriano J, Silbiger S, Schlöndorff D: Intracellular glutathione influences collagen generation by mesangial cells. Kidney Int 1994;46:388-395.

23 Boesgaard S, Aldershvile J, Poulsen HE, Christensen S, Dige-Petersen H, Giese J: N-acetylcysteine inhibits angiotensin converting enzyme in vivo. J Pharmacol Exp Ther 1993;265: 1239-1244.

24 Loske C, Neumann A, Cunningham A, Nichol K, Schinzel R, Riederer P, Munch G: Cytotoxicity of advanced glycation endproducts is mediated by oxidative stress. J Neural Transm 1998;105:1005-1015.

25 Hultberg B, Andersson A, Masson P, Larson M, Tunek A: Plasma homocysteine and thiol compound fractions after oral administration of N-acetylcysteine. Scand J Clin Lab Invest 1994;54:417-422.

26 Burgunder J, Varriale A, Lauterburg B: Effect of N-acetylcysteine on plasma cysteine and glutathione following paracetamol administration. Eur J Clin Pharmacol 1989;36:127-131.

27 Otieno M, Anders M: Cysteine S-conjugates activate transcription factor NF-kappa B in cultured renal epithelial cells. Am J Physiol 1997;273(1 Pt 2):F136-F143.
28 Khachigian L, Collins T, Fries J: N-acetylcysteine blocks mesangial VCAM-1 and NF-kappa B expression in vivo. Am J Pathol 1997;151: 1225-1229.

29 Tariq M, Morais C, Sobki S, Al Sulaiman M, Al Khader A: N-acetylcysteine attenuates cyclosporin-induced nephrotoxicity in rats. Nephrol Dial Transplant 1999;14:923-929.

30 Pincemail J, Defraigne JO, Detry O, Franssen C, Meurisse M, Limet R: Ischemia-reperfusion injury of rabbit kidney: Comparative effects of desferrioxamine and $\mathrm{N}$-acetylcysteine as antioxidants. Transplant Proc 2000;32:475-476.

31 Rangan G, Wang Y, Tay Y, Harris D: Inhibition of nuclear factor-kappaB activation reduces cortical tubulointerstitial injury in proteinuric rats. Kidney Int 1999;56:118-134.

32 Kleinveld H, Demacker P, Stalenhoef A: Failure of $\mathrm{N}$-acetylcysteine to reduce low-density lipoprotein oxidizability in healthy subjects. Eur J Clin Pharmacol 1992;43:639-642.

33 Safirstein R, Andrade L, Vieira JM: Acetylcysteine and nephrotoxic effects of radiographic contrast agents - a new use for an old drug. N Engl J Med 2000;343:210-212.

34 Heyman SN, Goldfarb M, Shina A, Karmeli F, Rosen S: N-acetylcysteine ameliorates renal microcirculation: Studies in rats. Kidney Int 2003;63:634-641.

35 Zhang H, Spapen H, Nguyen DN, Rogiers P, Bakker J, Vincent JL: Effects of N-acetyl- $L$-cysteine on regional blood flow during endotoxic shock. Eur Surg Res 1995;27:292-300.

36 Jones A, Hynes W, MacGilchrist AJ: N-acetylcysteine (NAC) is a potent peripheral vasodilator (abstract). Gut 1994;35:S10.

37 Allaqaband S, Tumuluri R, Malik A, Gupta A, Volkert P, Shalev Y, Bajwa TK: Prospective randomized study of $\mathrm{N}$-acetylcysteine, fenoldopam, and saline for prevention of radiocontrast-induced nephropathy. Catheter Cardiovasc Interv 2002;57:279-283.

38 Alonso A, Lau J, Jaber L, Weintraub A, Sarnak MJ: Prevention of radiocontrast nephropathy with $\mathrm{N}$-acetylcysteine in patients with chronic kidney disease: A meta-analysis of randomized controlled trials (abstract). J Am Soc Nephrol 2003; 14:282.

39 Findlay A, Dwomoa A: Acetylcysteine in the prevention of contrast nephropathy: Metaanalysis of randomised trials (abstract). J Am Soc Nephrol 2003;14:454.

40 Pannu N, Manns B, Lee H, Tonelli M: Systematic review of the impact of $\mathrm{N}$-acetylcysteine on contrast nephropathy (abstract). J Am Soc Nephrol 2003;14:282.

41 Fishbane S, McCullough PA, Rudnick M: Systematic review of $\mathrm{N}$-acetylcysteine (NAC) in the prevention of contrast media-induced nephropathy (CIN) (abstract). J Am Soc Nephrol 2003; $14: 281$.

42 Emch TM, Haller NA: A randomized trial of prophylactic acetylcysteine and theophylline compared with placebo for the prevention of renal tubular vacuolization in rats after iohexol. Acad Radiol 2003;10:514-519. 
43 Baker CS, Wragg A, Kumar S, De Palma R, Baker LR, Knight CJ: A rapid protocol for the prevention of contrast-induced renal dysfunction: The RAPPID study. J Am Coll Cardiol 2003;41:2114-2118.

44 Rickli H, Benou K, Ammann P, Fehr T, Brunner-La Rocca HP, Petridis H, Riesen W, Wüthrich RP: Time course of serial cystatin C levels in comparison with serum creatinine after application of radiocontrast media. Clin Nephrol 2004;61:98-102.

45 Levey AS, Bosch JP, Lewis JB, Greene T, Rogers N, Roth D: A more accurate method to estimate glomerular filtration rate from serum creatinine: A new prediction equation. Ann Intern Med 1999;130:461-470.
46 Mussap M, Dalla Vestra M, Fioretto P, Saller A, Varagnolo M, Nosadini R, Plebani M: Cystatin $\mathrm{C}$ is a more sensitive marker than creatinine for the estimation of GFR in type 2 diabetic patients. Kidney Int 2002;61:1453-1461.

47 Coll E, Botey A, Alvarez L, Poch E, Quinto L, Saurina A, Vera M, Piera C, Darnell A: Serum cystatin $\mathrm{C}$ as a new marker for noninvasive estimation of glomerular filtration rate and as a marker for early renal impaiment. Am J Kidney Dis 2000;36:29-34.

48 Le Bricon T, Thervet E, Froissart M, Benlakehal M, Bousquet B, Legendre C, Erlich D: Plasma cystatin $\mathrm{C}$ is superior to 24 -h creatinine clearance and plasma creatinine for estimation of glomerular filtration rate 3 months after kidney transplantation. Clin Chem 2000;46(8 Pt 1):1206-1207.
49 Filler G, Priem F, Lepage N, Sinha P, Vollmer I, Clark H, Keely E, Matzinger M, Akbari A, Althaus H, Jung K: Beta-trace protein, cystatin $\mathrm{C}$, beta(2)-microglobulin, and creatinine compared for detecting impaired glomerular filtration rates in children. Clin Chem 2002;48:729_ 736.

50 Genet S, Kale RK, Baquer NZ: Effects of free radicals on cytosolic creatinine kinase activities and protection by antioxidant enzymes and sulfhydryl compounds. Mol Cell Biochem 2000;210:23-28. 